\title{
Dissociated vertical deviation: evidence of abnormal visual pathway projection
}

\author{
B. ANNE FITZGERALD AND FRANCIS A. BILLSON \\ From the University of Sydney Department of Clinical Ophthalmology, Sydney, Australia
}

SUMMARY Abnormalities in visually evoked responses have been used to demonstrate abnormal optic nerve fibre projections in human albinos, who have anomalous nystagmoid movements. Using visually evoked potentials we tested the hypothesis that patients with dissociated vertical deviation (another group with anomalous nystagmoid movements) may have abnormal visual evoked responses similar to those of albinos. Patients with dissociated vertical deviation (both unilateral and alternating), patients with congenital esotropia without dissociated vertical deviation, and normal subjects were investigated. The results showed a very high incidence of abnormal visual evoked responses in patients with dissociated vertical deviation, while recordings from the other groups were normal. As well as suggesting the possibility of abnormalities of optic nerve fibre projections, the visual evoked responses showed a marked increase in latency in all patients with dissociated vertical deviation. These results occurred regardless of the visual acuity or amblyopia of the eyes tested. The possible existence of abnormal pathway projection in the presence of dissociated vertical deviation is discussed.

Dissociated vertical deviation (DVD) is an intriguing and ill understood vertical motor disturbance in which either eye elevates with extorsion and latent nystagmus when the amount of light entering the eye is reduced. ${ }^{12}$

DVD may be an isolated phenomenon in patients in whom some degree of binocular vision is apparent. Most frequently it is seen in association with congenital esotropia, though the DVD is not seen until a number of years later. Occasionally the deviation is thought to occur unilaterally, and some authors say that although it may be asymmetrical it is almost always a bilateral phenomenon. ${ }^{3}$

Recent studies ${ }^{4}$ have reported that patients with DVD also have anomalous optokinetic nystagmus (OKN) when the optokinetic drum is rotated nasotemporally. Another group of patients with abnormal monocular $\mathrm{OKN}$ and weak or absent binocular vision are albinos. In 1974 Creel et al. ${ }^{5}$ tested albinos with checkerboard visual evoked responses (VERs) and found that up to $70 \%$ had abnormal temporal retinal fibre projections. This work has been supported by other publications. ${ }^{67} \mathrm{~A}$ similar abnormality has been

Correspondence to B. Anne Fitzgerald, Department of Clinical Ophthalmology, Sydney Eye Hospital, Sir John Young Crescent, Sydney, NSW 2011, Australia. reported in cats with Chediak-Higashi syndrome ${ }^{8}$ (CHS) tested by means of autoradiography and in man with congenital nystagmus" by means of flash VERs.

As there are several similarities between albinos, patients with latent nystagmus, cats with $\mathrm{CHS}$, and patients with DVD (including strabismus, nystagmus, and anomalous OKN), a group of patients with DVD were investigated to see if they also have an abnormality similar to the VER abnormality found in albinos. The controls were a group of normal subjects, and a group of patients with congenital strabismus who did not have DVD or nystagmus were also investigated. In view of Mein's ${ }^{4}$ work it was also decided to observe clinically $\mathrm{OKN}$ in all patients tested with VER.

\section{Materials and methods}

The three groups of patients examined were as follows.

Group I consisted of 12 ophthalmologically normal subjects. Each had normal binocular vision and visual acuity of $6 / 6$ or better. Only the right eye was tested.

Group II consisted of 12 patients with congenital strabismus with a positive history of squint before 6 months of age. Both eyes were tested. 

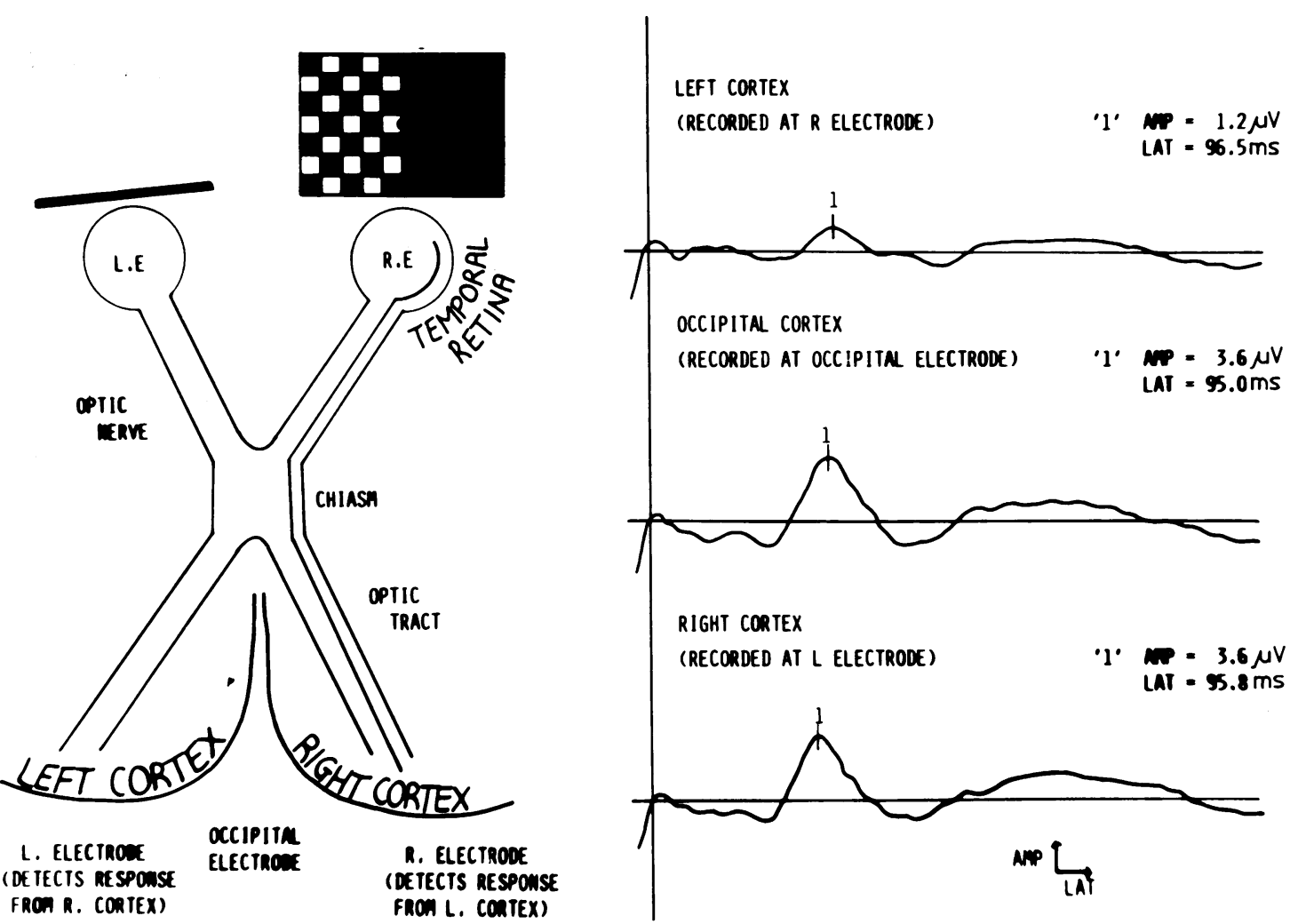

Fig. 1 Normal subject, right eye; temporal hemiretina stimulated. The maximal response is recorded from the ipsilateral occipital lobe recording electrode.

Group IIIa consisted of 14 patients with unilateral DVD (two of these patients had weak binocular functions). Both eyes were tested. Group IIIb: consisted of 17 patients with alternating DVD. Both eyes were tested.

Half-field checkerboard VERs were performed monocularly on every patient. When the patient was seated $57 \mathrm{~cm}$ from the checkerboard $1 \mathrm{~cm}$ checks of $100 \%$ contrast (which subtended $1^{\circ}$ at the nodal point) were projected onto a screen and reversed twice per second $(1 \mathrm{~Hz})$. The checkerboard stimuli had a mean average luminance of 95 candelas $/ \mathrm{m}^{2}$. Recordings were taken from three active electrodes. Thus the relative contributions of optic nerve pathways to the visual cortex could be determined. One electrode was placed in the midline $5 \mathrm{~cm}$ above the inion. Two other electrodes were placed $5 \mathrm{~cm}$ laterally on either side of the occipital electrode. Recordings were taken individually from these electrodes, so that they detected responses arriving at the cortex from separate stimulation of nasal and temporal retina. The reference electrode was placed on the forehead and the earth electrode on the ear. Fixation control was dependent on a careful explanation to the patient, and a trial VER was performed to ensure that the instructions about fixation were understood. Where doubt about co-operation existed, the patient's fixation was monitored visually by an observer.

Clinical observations of OKN were made. A 17.5 $\mathrm{cm}$ diameter $\mathrm{OKN}$ drum with $2 \mathrm{~cm}$ stripes separated by $7 \mathrm{~cm}$ was rotated at an average velocity of $0.23 \pm$ 0.02 revolutions per second. The stripes subtended $3.5^{\circ}$ at the nodal point. The drum was held $33 \mathrm{~cm}$ from the patient. Qualitative OKN responses were observed when the drum was rotated nasotemporally $(\mathrm{N} \rightarrow \mathrm{T})$ and temporonasally $(\mathrm{T} \rightarrow \mathrm{N})$ in front of each eye in turn. Both slow and fast phases of OKN were observed. In patients with latent nystagmus the OKN drum was rotated so that stripe movement was in phase' with the slow phase of latent nystagmus.

OKN was considered normal when there was a jerk nystagmus with a constant velocity slow phase in the direction of drum rotation and the fast phase in the opposite direction.

In this paper the following conventions have been observed. Recordings at the ipsilateral occipital lobe 


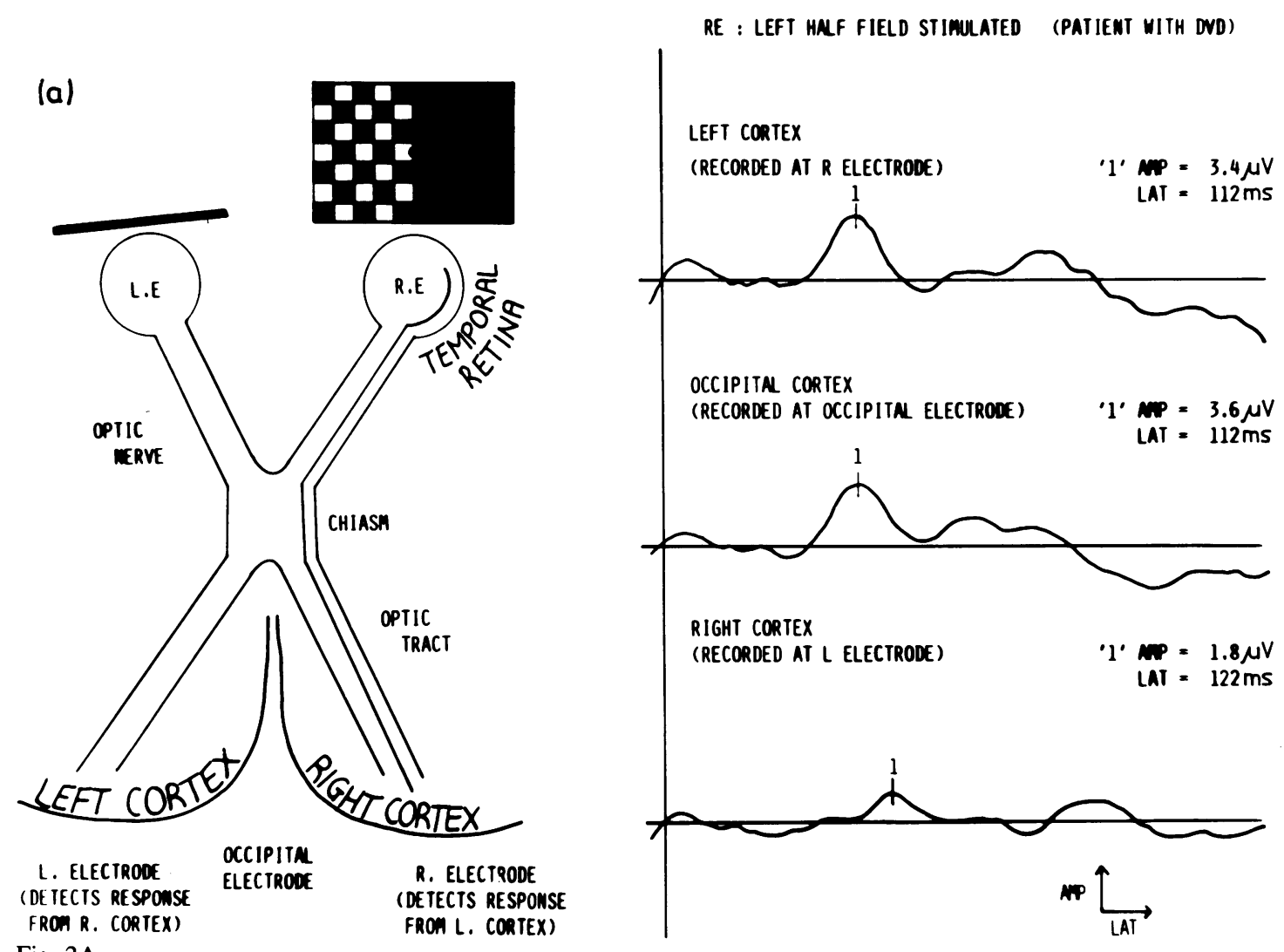

Fig. 2A

Fig. 2 Right eye; temporal hemiretina stimulated. Patient with dissociated vertical deviation. A: When temporal retina is stimulated $(R E)$ the maximal response is recorded from the contralateral occipital lobe recording electrode. B: In patients with dissociated vertical deviation temporal retinal fibres may project incorrectly and decussate at the chiasm; hence the 'normal' response recorded from the contralateral occipital lobe recording electrode (represented by dashed line).

electrode, for example in the right eye, are as follows: When temporal hemiretina is maximally stimulated, nerve fibre projections normally carry the response to the right visual cortex. This response is recorded at the electrode on the left side of the occipital cortex. (This paradoxical recording is in keeping with findings of Barrett, Blumhardt, and colleagues $\left.{ }^{10} 11\right)$. In this instance the left electrode is referred to as the ipsilateral occipital lobe recording electrode, as it is ipsilateral to the visual half field in which the checkerboard is being presented (Fig. 1). It records the response from the right occipital cortex. 'Mean' amplitudes quoted in this section refer to amplitudes for all patients in the group.

\section{Results}

GROUP I: NORMAL SUBJECTS

$V E R$ results. Amplitude: when the temporal hemiretina was stimulated the amplitude recorded (mean
$4.01 \mu \mathrm{V})$ at the ipsilateral occipital lobe recording electrode-i.e., the electrode ipsilateral to the half field in which the checkerboard was presented-was greater than the amplitude recorded (mean $1 \cdot 13 \mu \mathrm{V}$ ) at the contralateral occipital lobe recording electrode -i.e., the electrode contralateral to the half field in which the checkerboard was presented (Fig. 1). As projections from nasal retina decussate at the chiasm, the situation was reversed in all patients when the nasal hemiretina was stimulated. Hence the tracing at the contralateral occipital lobe recording electrodei.e., the electrode contralateral to the half field in which the checkerboard was presented-was greater than the amplitude at the other electrode. This was the characteristic response for all patients in group I. (In this study, as in most others, there was considerable variation in 'normal' amplitudes between subjects.)

Latency: when the temporal hemiretina was stimulated, the average latency at the ipsilateral occipital 
RE : LEFT HML FIELD STIMULATED (PATIENT MITH DVD)

(b)

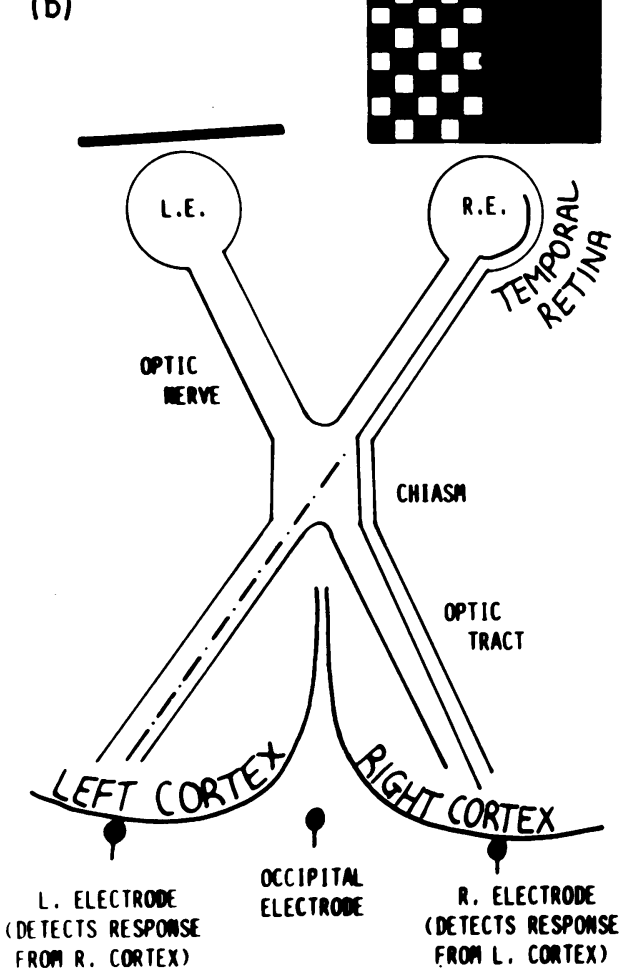

Fig. 2B

recording electrode was $96.6 \mathrm{~ms}$. The latency from the contralateral occipital lobe recording electrode was slightly increased to $100 \cdot 22 \mathrm{~ms}$ (Fig. 1).

OKN results. When observing OKN responses from normal subjects with the unaided eye we did not find directional asymmetry in any subject. Both slow and fast phases of OKN appeared normal in both eyes of each subject.

GROUP II: CONGENITAL STRABISMUS

$V E R$ results. Amplitude: when the temporal hemiretina was stimulated, the results were similar to those in group I in $91.8 \%$ of the patients with congenital strabismus. All the patients in this group showed the same response as patients in group I when the nasal hemiretina was stimulated.

Latency: in all patients with congenital strabismus a slight increase in latencies was recorded at the occipital cortex (mean 109.22 ms).

OKN results. $25 \%$ had anomalous $\mathrm{OKN}$ responses (similar to the abnormalities to be discussed in patients with DVD) when the drum was rotated nasotemporally. Only one case was observed to have abnormal temporonasal OKN.

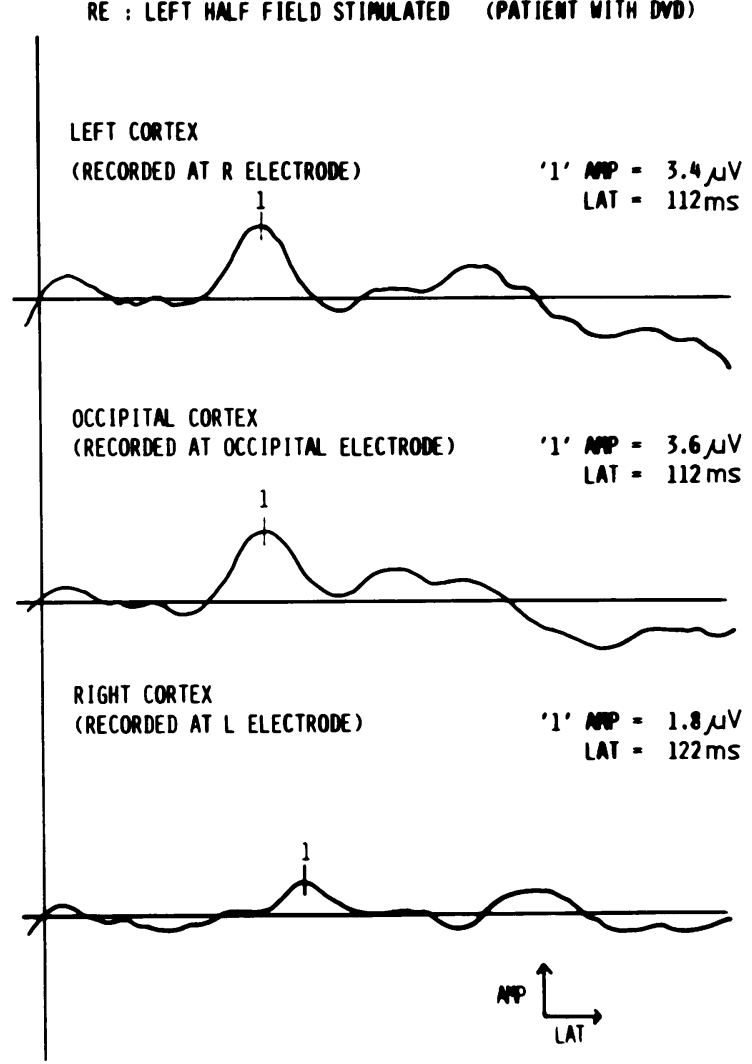

GROUP III: PATIENTS WITH DVD

$V E R$ results. Unilateral DVD: Amplitude: contrary to the results recorded in normal subjects and patients with congenital strabismus, when the temporal hemiretina was stimulated in the eye with the DVD, the amplitude recorded (mean $3 \cdot 12 \mu \mathrm{V}$ ) at the ipsilateral occipital lobe recording electrode-i.e., the electrode ipsilateral to the half field in which the checkerboard was presented-was smaller than the amplitude recorded (mean $3.08 \mu \mathrm{V})$ at the contralateral occipital lobe recording electrode-i.e., the electrode contralateral to the half field in which the checkerboard was presented-in $78.5 \%$ of the cases (Fig. 2).

In the eye without the clinical characteristics of DVD the abnormality occurred in only four of the 14 cases.

When the nasal hemiretina was stimulated the responses were similar to those recorded in normal subjects with the tracing at the contralateral occipital lobe recording electrode -i.e., the electrode contralateral to the half field in which the checkerboard was presented-being greater in amplitude (mean 3.10 $\mu \mathrm{V}$ ) that the amplitude of the tracing (mean $2.40 \mu \mathrm{V}$ ) at the ipsilateral occipital lobe recording electrode- 
i.e., the electrode ipsilateral to the stimulated half field-in $72 \%$ of the cases. The other $28 \%$ showed an abnormality similar to that noted when temporal retina was stimulated.

Alternating DVD: Amplitude: a similar abnormality to that in the unilateral DVD group was obtained in $76.4 \%$ with alternating DVD when the temporal hemiretina was stimulated. The mean amplitude recorded at the ipsilateral occipital lobe recording electrode was $3.52 \mu \mathrm{V}$. This was again smaller in amplitude than the response from the contralateral occipital lobe recording electrode (mean $4.08 \mu \mathrm{V}$ ).

When the nasal hemiretina was stimulated, $64 \cdot 7 \%$ of the patients showed responses similar to those obtained from normal subjects and patients with congenital strabismus.

Latency: in all patients with DVD there was an increase in the latency of the $P_{100}$. This increase occurred when both nasal and temporal hemiretina were stimulated. It was most marked at the ipsilateral occipital lobe recording electrode.

When the results of the unilateral and alternating DVD groups were combined, $77.4 \%$ of the patients showed abnormally high amplitude recordings at the contralateral occipital lobe recording electrode when the temporal hemiretina were stimulated.

$O K N$ results. Unilateral DVD: $71.4 \%$ showed abnormal OKN when the drum was rotated $\mathrm{N} \rightarrow \mathrm{T}$ in front of the affected eye. The abnormalities included absence of fast phase, jerky slow phase, and a slower velocity OKN than the OKN observed when the drum was rotated the other way.

Alternating DVD; $76 \cdot 5 \%$ had similarly abnormal responses when the drum was rotated $\mathrm{N} \rightarrow \mathrm{T}$.

All but three of the patients in group III were observed to have normal $\mathrm{T} \rightarrow \mathrm{N}$ OKN.

For a summary of all results see Table 1 .

\section{Discussion}

The abnormality in VER recordings which occurs in $77 \cdot 4 \%$ of the patients with DVD when the temporal hemiretina is stimulated is similar to the abnormality reported to be found in $70 \%$ of human albinos. ${ }^{56}$ In

Table 1 Summary of results

\begin{tabular}{lll}
\hline Patient group & $\begin{array}{l}\text { Normal temporal retinal } \\
\text { fibre projection }\end{array}$ & $\begin{array}{l}\text { Normal } \\
\text { OKN }\end{array}$ \\
\hline I. Normal subjects & $\mathrm{Y}$ & $\mathrm{Y}$ \\
II. Congenital strabismus & $\mathrm{Y}$ & $\mathrm{Y}$ \\
IIIa. Unilateral DVD & $\mathrm{X}$ & $\mathrm{X}$ \\
IIIb. Alternating DVD & $\mathrm{X}$ & $\mathrm{X}$ \\
\hline
\end{tabular}

$\mathrm{Y}=$ yes. $\mathrm{X}=$ no. albinos the abnormality in VER recordings is thought to occur because temporal retinal fibres project incorrectly by crossing over at the chiasm. Therefore the results of this study lend support to the possibility that a similar situation may occur in patients with DVD. Temporal retinal fibre projections which usually pass directly to the visual cortex on the same side may project incorrectly and cross over at the chiasm, thus terminating in the opposite (or contralateral) visual cortex. As yet there is no histological confirmation of abnormal retinostriate projections comparable to those of the albino study.

This finding occurred in a high percentage of patients only in the group with DVD. It was not present at all in the normal subjects and was only evident in $8 \%$ of the patients with congenital strabismus. Thus in this study evidence to suggest abnormal temporal retinal fibre projections is not present in strabismic patients without DVD. This finding is in keeping with a study by McCormack, ${ }^{12}$ who studied strabismic patients with normal ocular pigment and concluded that there was no visual pathway anomaly.

Abnormalities in temporal retinal striate projection have been demonstrated in albinos, ${ }^{5}$ Chediak-Higashi syndrome,${ }^{8}$ chiasmal lesions,${ }^{13}$ congenital nystagmus, ${ }^{9}$ and now in DVD. The possibility that DVD and congenital nystagmus may be included in the group with retinostriate projection abnormalities raises an interesting question in relation to the association of retinal pigment epithelium and its influences concerned with the formation of optic nerve projection.

In 1980 Silver and Sapiro ${ }^{14}$ histologically examined a series of embryos at stages when ocular pigment and outgrowing axons first became apparent. They stated that there is a region of the primitive stalk that becomes transiently pigmented prior to and during migration of the pioneer optic axons and that outgoing neurons avoid this melanotic tissue. They further suggested that melanin-producing stalk cells may play a role in controlling the topographic patterning of optic fibres within the developing nerve by preventing axonal growth cones from entering the territory. In albinos the absence of melanin in the retinal pigment epithelium may well be a marker as to the state of pigment in the distal eye stalk during development. In patients with DVD and congenital nystagmus, where retinal pigment epithelium appears normal, the possibility that abnormalities in the pigment of the developing distal eye stalk may occur in isolation must not be excluded; alternatively the evidence for the projection abnormality may be caused by other mechanisms.

In the unilateral DVD group $72 \%$ showed VER recording abnormalities in only one eye, indicating that DVD may indeed occur unilaterally in some 
cases. It is of interest to note that $28 \%$ had abnormal VERs when temporal retina was stimulated in the eye in which the DVD was not clinically manifest. These four cases will be followed up to see if DVD develops in the other eye when the patient is older.

The fact that DVD may be asymmetrical (verified by asymmetrical VER tracings) or unilateral, supports the current attitudes to asymmetrical surgery for symptomatic DVD in this Department. Asymmetrical surgery is performed where indicated (Billson and Fitzgerald, in preparation), and the results compare favourably with those of other reported series.

If the VER abnormalities recorded in DVD were to be explained on the same basis as those seen in albinos, then an abnormality of temporal fibre projection would provide part of the explanation of the clinical finding of weak or no binocular vision in patients with $\mathrm{DVD}^{4}$ and albinism. The abnormal fibre projection would cause disruption of the ordered structure of the lateral geniculate nucleus (LGN). If a large enough number of temporal retinal fibres are misdirected through the chiasm and the LGN to the cortex, then there could not be normal duplication of visual fields; hence no normal binocular association of the cortex.

Confirmation of the findings of the abnormality of retinostriate projections in the presence of a high incidence of latent nystagmus and good visual acuity would raise interesting questions, particularly in respect of abnormalities of sensory pathways, possibly extrageniculate as the basis of nystagmus that might otherwise give the clinical appearance of being motor.

The increase in latency found in VERs recorded from temporal retinal stimulation of patients with DVD was most marked in cases where latent nystagmus was most evident. This finding awaits further interpretation. The possible use of more channels may reveal a latency change related to a wave form change. ${ }^{15}$

The results of OKN testing have confirmed Mein's ${ }^{4}$ finding that anomalous $\mathrm{N} \rightarrow \mathrm{T}$ induced $\mathrm{OKN}$ may occur in patients with DVD. In this study there is a high correlation between abnormalities in VERs and nasotemporally induced $\mathrm{OKN}$. This suggests that monocular OKN tests should be performed as a routine on children with strabismus, especially those with congenital esotropia who are still young enough to develop DVD. Perhaps those with anomalous OKN might be regarded as at risk of developing DVD. The possibility of abnormality in temporal retinal fibre projection occurring in the majority of patients with DVD would add another clinical association to the syndrome of DVD.

The authors would like to thank Dr Jan M. Provis and Dr Vaegan for their help in data analysis, and the Orthoptic Department, Sydney Eye Hospital.

\section{References}

1 Bielschowsky A. Über aneborene und erworbene Blickfelderweiterungen. Ber Dtsch Ophthalmol Ges 1911; 37: 192-4.

2 Anderson JR. Ocular vertical deviations and nystagmus. In: Burian HM, von Noorden MD, eds. Binocular vision and ocular motility. St Louis: Mosby, 1974: 319-26.

3 Parkes MM. Dissociated hyperdeviation in ocular motility and strabismus. New York: Harper and Rowe, 1975: 149.

4 Mein J. The OKN response and binocular vision in early onset strabismus. Aust Orthopt J 1983; 20: 13-8.

5 Creel D, Wikop CJ, King RA. Asymmetric visually evoked potentials in human albinos; evidence for visual system anomalies. Invest Ophthalmol Visual Sci 1974; 13: 430-40.

6 Jay B, Carroll W. Albinism: recent advances. Trans Ophthalmol Soc UK 1980; 100: 467-71.

7 Creel D, Spekreijse H, Retis D. Visual evoked potential (VEP) methods of detecting misrouted optic projections. Doc Ophthalmol Proc Ser 1981; 27: 157-65.

8 Creel D, Collier LL, Leventhal AG, Conlee JW, Prieur DJ. Abnormal retinal projections in cats with Chediak-Higashi syndrome. Invest Ophthalmol Visual Sci 1982; 23: 798-801.

9 Meienberg O, Hemphill G, Rosenberg M, Hoyt W. Visually evoked response asymmetries in a family with congenital nystagmus. Possible evidence of abnormal visual projections. Arch Neurol 1980; 37: 697-8.

10 Barrett G, Blumhardt L, Halliday A, Halliday E, Kriss A. A paradox in the lateralisation of the visual evoked response. Nature 1976; 261: 253-7.

11 Blumhardt L, Halliday A. Hemisphere contributions to the composition of the pattern-evoked potential waveform. Exp Brain Res 1979; 36: 53-69.

12 McCormack GL. Electrophysiological evidence of normal optic nerve fibre projection in normally pigmented squinters. Invest Ophthalmol Visual Sci 1975; 14: 931-5.

13 Halliday AM, Halliday E, Kriss A, McDonald WI, Mushin J. The pattern-evoked potential in compression of the anterior visual pathways. Brain 1976; 99: 357-74.

14 Silver J, Sapiro J. The role of pigmented epithelia during maturation of the optic nerve. ARVO abstracts $1980 ; 3$.

15 Carroll WM, Halliday AM, Kriss A. Improvements in the accuracy of pattern visual evoked potentials in the diagnosis of visual pathway disease. Neuro-Ophthalmology 1982; 2: 237-53. 